\title{
Etude de l'influence des défauts de petite taille sur le comportement à rupture avec le modèle de Dugdale régularisé
}

\author{
Hichème Ferdjani* — Med Zaim Khelifi* \\ Jean-Jacques Marigo** \\ * Université Saad Dahlab, Département de Mécanique \\ Route de Soumaa, Blida, Algérie \\ h_ferdjani@yahoo.fr, k_zaim@yahoo.fr. \\ ** Institut Jean Le Rond D'Alembert (UMR-CNRS 7607), Université Paris 6 \\ 4, Place Jussieu case 162, F-75252 Paris cedex 5 \\ marigo@lmm.jussieu.fr
}

RÉSUMÉ. Le but du travail est de montrer, dans le cadre de la mécanique de la rupture avec le modèle de Dugdale régularisé des forces cohésives, que les défauts de petite taille devant la longueur caractéristique du matériau ont pratiquement peu d'influence sur les capacités de résistance d'une structure. On traite pour cela deux exemples : le cas d'une plaque préfissurée, puis le cas d'une plaque contenant une cavité circulaire. Les calculs sont effectués avec la méthode des éléments finis.

ABSTRACT. The goal of this work is to prove that, within the framework of Fracture Mechanics with the regularized Dugdale's model of cohesive forces, the defects the size of which are small compared to the material characteristic length are practically without influence on the limit loads of structures. For that, we treat two examples : the case of a precracked plate, then the case of a plate with a circular hole. The calculations are made with the finite element method.

MOTS-CLÉS : rupture, modèle de Dugdale régularisé, effet d'échelle, charges limites.

KEYWORDS: fracture, Regularized Dugdale's model, scale effect, limit loads.

DOI:10.3166/REMN.17.481-493 @ 2008 Lavoisier, Paris 


\section{Introduction}

Les modèles de rupture de forces cohésives présentent l'avantage, sur le modèle de Griffith, de rendre compte de l'amorçage de fissure dans une structure saine en termes d'un critère en contraintes, (cf. par exemple Del Piero, 1999, Del Piero et al., 2001, Charlotte et al., 2000, Charlotte et al., 2006, Laverne et al., 2004). De façon générale, les modèles de forces cohésives sont de plus en plus employés et il est donc nécessaire de connaître de mieux en mieux leurs propriétés pour pouvoir les utiliser à bon escient. En particulier les effets d'échelle qui les accompagnent du fait de la présence d'une longueur caractéristique sont mal connus. Il ne s'agit évidemment pas ici de valider le modèle des forces cohésives, ni de voir pour quel type de matériau il peut être utilisé, mais de voir s'il est assez robuste pour que, grâce aux effets d'échelle qu'il induit, la réponse des structures ne soit pas sensible à des défauts de petite taille. Ce résultat a été obtenu par Ferdjani et al. (2006a), Ferdjani et al. (2006b) et Ferdjani et al. (2007), dans le cas du modèle de Dugdale (cf. Dugdale, 1960), pour le cas particulier d'une plaque contenant une fissure ou une cavité circulaire sollicitée en traction simple. L'objectif de cette étude est la généralisation de ce résultat à d'autres modèles de forces cohésives.

Le modèle étudié dans ce travail, et appelé modèle de Dugdale régularisé, est une combinaison entre le modèle des zones cohésives continuums (cf. Xie et al., 2006) et le modèle de Dugdale. Ce modèle ajoute au modèle de Dugdale une zone cohésive continuum (ZCC) dans la partie saine du matériau qui se situe sur le trajet de la fissure. La loi de comportement, donnant la relation entre les forces cohésives et l'ouverture des lèvres de la fissure, en supposant que l'ouverture se fait en mode I pur, est représentée sur la figure 1.

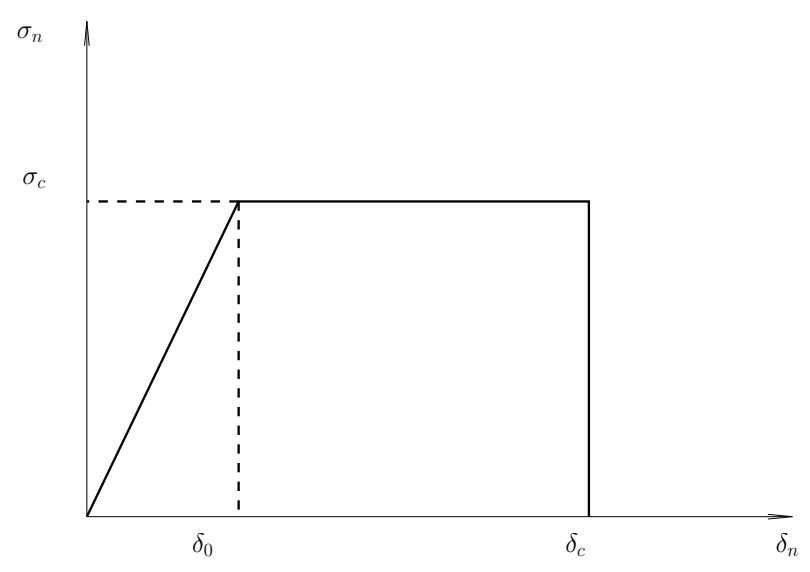

Figure 1. Loi de comportement dans le modèle de Dugdale régularisé 
Dans la figure 1, $\delta_{n}$ désigne la discontinuité du déplacement normal, $\sigma_{n}$ désigne la contrainte normale d'interaction entre les lèvres de la fissure, $\sigma_{c}$ désigne la contrainte critique du matériau, $\delta_{c}$ désigne la longueur caractéristique du modèle, et $\delta_{0}$ désigne le saut critique de décohésion. La fissure est donc divisée en trois zones représentées sur la figure 2.

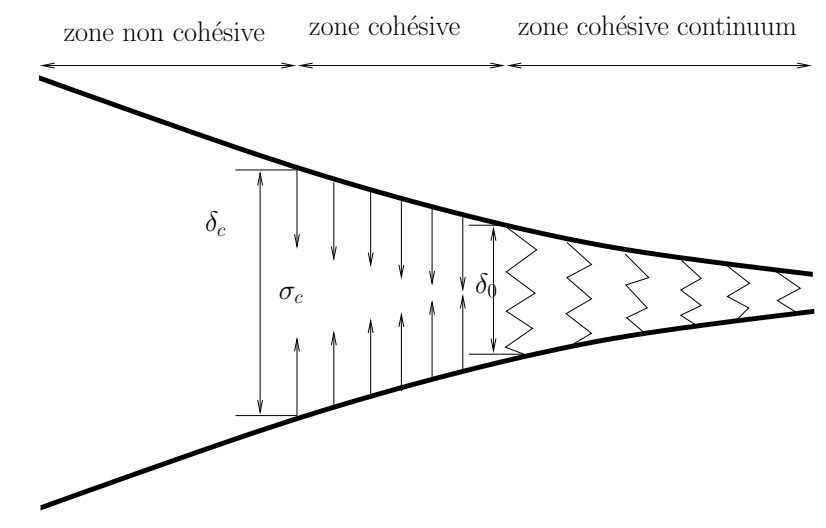

Figure 2. Les différentes zones de la fissure dans le modèle de Dugdale régularisé

Ce modèle a une origine et une justification purement phénoménologiques. Le lecteur intéressé par une justification micromécanique de ce type de modèle peut se référer à (Budiansky et al., 1995) où, à partir d'une analyse simplifiée, les auteurs arrivent à construire un modèle cohésif effectif pour des composites contenant une fissure principale matricielle pontée par des fibres partiellement décollées.

Notons que ce type de modèle possède le défaut majeur de présenter une contrainte d'amorçage nulle, ce qui veut dire que le déplacement est discontinu dès la mise en charge à travers toutes les surfaces autorisées. Il ne faut donc l'utiliser que dans des situations où le trajet de fissuration est connu a priori. C'est dans cet esprit que ce type de modèle a été introduit dans (Needleman, 1987) avant d'être repris abondamment par la suite, (cf. par exemple Needleman, 1990, Tvergaard, 1990, Needleman, 1992, Chaboche et al., 2001, Roe et al., 2002). D'un point de vue numérique, l'introduction d'une phase «élastique » par rapport à la phase «rigide » de Dugdale permet d'avoir une relation univoque entre ouverture et contrainte cohésive. Nous allons donc l'utiliser dans un cadre où il est pertinent, i.e. en se donnant le trajet de fissuration. Le principal objectif de ce papier est de voir si l'introduction d'une phase élastique modifie les conclusions obtenues pour le modèle de Dugdale.

Du fait de la présence d'une longueur caractéristique $\delta_{c}$ dans le modèle des forces cohésives, les effets d'échelle sont importants. La charge de rupture dépend de façon essentielle du rapport entre la taille du défaut et la longueur caractéristique $\delta_{c}$. Un résultat majeur serait de montrer que, quelle que soit la forme du défaut, la charge de rupture tend vers la contrainte critique $\sigma_{c}$ lorsque la taille du défaut tend vers 0 , 
à longueur caractéristique fixée (ou de façon équivalente, lorsque la longueur caractéristique $\delta_{c}$ tend vers l'infini, à taille du défaut fixée). Ceci signifierait qu'avec le modèle des forces cohésives, les structures sont insensibles aux petits défauts et se comportent comme des structures saines, et ce bien qu'elles développent des zones d'amorçage avant rupture. On se contentera d'étudier ici les deux cas extrêmes et de montrer qu' une plaque en traction simple est insensible à un petit défaut de type fissure ou cavité circulaire justement en raison des effets d'échelle.

L'article est organisé comme suit. Dans la section 2, le problème est posé. Dans la section 3, on le résout numériquement par la méthode des éléments finis dans le cas d'une fissure préexistante et d'une cavité circulaire. On synthétise les résultats obtenus et on conclut dans la section 4 .

\section{Position du problème}

On considère un milieu bidimensionnel carré $\Omega=(-L,+L)^{2}$ contenant un défaut centré $\mathcal{D}$ et soumis sur les faces supérieure $x_{2}=+L$ et inférieure $x_{2}=-L$ à une traction uniforme d'intensité $\sigma_{\infty}$ croissante depuis 0 . Le défaut considéré $\mathcal{D}$ sera soit une fissure préexistante perpendiculaire à l'axe de traction et de longueur $2 \ell_{0}$, soit un trou circulaire de rayon $\ell_{0}$ (figure 3 ). L'etude est effectuée dans le cadre des contraintes planes. Le matériau est linéairement élastique, homogène et isotrope, de tenseur de rigidité $\mathbf{A}$, de module de Young $E$ et de coefficient de Poisson $\nu$.

Pour des raisons de symétrie, on supposera que la fissuration ne se développe que sur l'axe $x_{2}=0$ et de façon symétrique depuis les points $\left( \pm \ell_{0}, 0\right)$ du défaut initial. L'evolution de la fissuration suit la loi de Dugdale régularisé, autrement dit les lèvres $\left(-L,-\ell_{0}\right)$ et $\left(+\ell_{0},+L\right)$ (de l'axe $\left.x_{2}=0\right)$ peuvent comporter trois parties :

1) une zone $\Gamma_{n}=\left[-\ell_{c},-\ell_{0}\right] \cup\left[\ell_{0}, \ell_{c}\right]$, proche du défaut initial et dite zone non cohésive, où les lèvres de la fissure n'interagissent plus ;

2) une zone $\Gamma_{c}=\left[-\ell_{a},-\ell_{c}\right] \cup\left[\ell_{c}, \ell_{a}\right]$ dite zone cohésive où les lèvres de la fissure sont soumises à des forces cohésives de traction d'intensité constante $\sigma_{c}$;

3) une zone $\Gamma_{z}=\left[-L,-\ell_{a}\right] \cup\left[\ell_{a}, L\right]$, dite zone cohésive continuum (ZCC), où les forces d'interaction entre lèvres sont proportionnelles à la discontinuité du déplacement normal à travers la fissure.

Les limites entre ces trois zones sont les points $x_{1}= \pm \ell_{c}$ et $x_{1}= \pm \ell_{a}$. Les valeurs de $\ell_{a}$ et $\ell_{c}$ dépendent évidemment de la valeur de la charge $\sigma_{\infty}$. Compte tenu de la symétrie du problème, toutes les évolutions se feront en mode I pur. Notons que pour certaines valeurs de $\sigma_{\infty}$ certaines zones peuvent ne pas exister. On a donc en général $\ell_{a} \geq \ell_{c} \geq \ell_{0}$, les égalités étant possibles. Lors de la mise en charge $\left(\sigma_{\infty}=0\right)$, les conditions initiales sont $\ell_{a}=\ell_{c}=\ell_{0}$. Les différents critères d'apparition et de propagation de ces zones sont les suivants :

1) une ZCC apparaît dés que la contrainte $\sigma_{22}$ n'est plus nulle aux points $x_{1}= \pm \ell_{0}$ de l'axe $x_{2}=0$, c'est-à-dire dès la mise en charge ; 


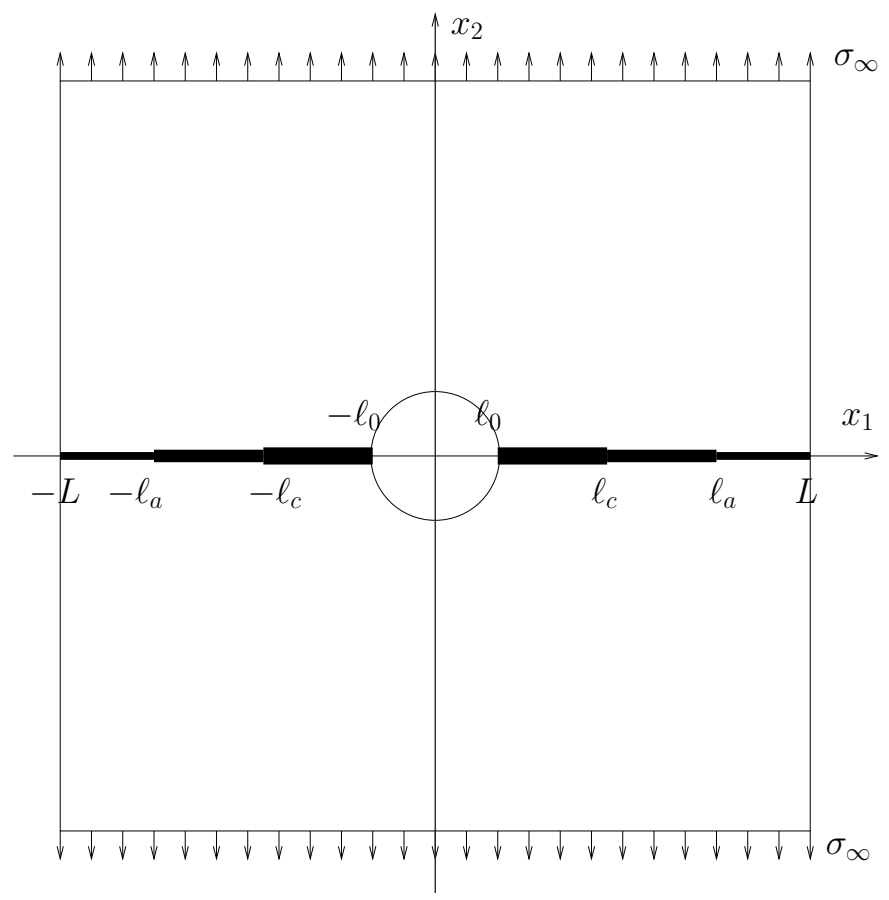

Figure 3. Géométrie de la plaque contenant le défaut centré avec les zones cohésive, non cohésive et la ZCC

2) une zone cohésive apparaît et dès lors $\ell_{a}>\ell_{c}=\ell_{0}$, lorsque l'ouverture atteint le saut critique de décohésion $\delta_{0}$ aux points $x_{1}= \pm \ell_{0}$;

3) les pointes $x_{1}= \pm \ell_{a}$ de la zone cohésive avancent de telle sorte que l'ouverture de la fissure $\llbracket u_{2} \rrbracket$ en ces points ne dépasse jamais le saut critique de décohésion $\delta_{0}$;

4) une zone non cohésive apparaît et dès lors $\ell_{a}>\ell_{c}>\ell_{0}$, lorsque l'ouverture atteint la valeur $\delta_{c}$ aux points $x_{1}= \pm \ell_{0}$;

5) les pointes $x_{1}= \pm \ell_{c}$ de la zone non cohésive avancent de telle sorte que l'ouverture ne dépasse jamais $\delta_{c}$ en ces points.

On peut a priori distinguer trois phases d'evolution de la fissuration lors du processus de chargement : 
1) phase élastique : $0 \leq \sigma_{\infty} \leq \sigma_{a}$. Durant cette phase, il n'y a que la ZCC qui apparaît et la réponse de la structure est purement élastique. Le champ des déplacements $u$ et le champ des contraintes $\sigma$ sont donc solution du problème élastique suivant :

$$
\left\{\begin{array}{ccc}
\text { div } \sigma=0 & \text { dans } & \Omega \backslash\left(\mathcal{D} \cup \Gamma_{z}\right) \\
\sigma=A \varepsilon(u) & \text { dans } & \Omega \backslash \mathcal{D} \\
\sigma n=k \llbracket u_{2} \rrbracket n & \text { sur } & \Gamma_{z} \\
\sigma n=0 & \text { sur } & \partial \mathcal{D} \\
\sigma n=0 & \text { sur } & x_{1}= \pm L \\
\sigma n=\sigma_{\infty} n & \text { sur } & x_{2}= \pm L
\end{array}\right.
$$

$n$ désignant la normale unitaire sortante au domaine avec défaut initial $\Omega \backslash \mathcal{D}$ et $k=\frac{\sigma_{c}}{\delta_{0}}$ désigne la rigidité de la ZCC.

Cette phase cesse lorsque l'ouverture maximale de la fissure atteint le saut critique de décohésion $\delta_{0}$. La valeur de la charge appliquée correspondante est appelée charge d'amorçage $\sigma_{a}$;

2) phase d'amorçage : $\sigma_{a}<\sigma_{\infty} \leq \sigma_{r}$. Durant cette phase, une zone cohésive se crée. Au fur et à mesure que l'on augmente le chargement, cette zone grandit et le saut de déplacement normal croît. Quand $\sigma_{\infty}$ est suffisemment proche de $\sigma_{a}$, l'ouverture $\llbracket u_{2} \rrbracket$ est partout inférieure à la valeur critique $\delta_{c}$. Par conséquent, les lèvres de la fissure ne comportent que deux zones : la zone cohésive et la ZCC. Durant cette phase le champ des déplacements $u$ et le champ des contraintes $\sigma$ sont donc solution du problème suivant :

$$
\left\{\begin{array}{ccc}
\operatorname{div} \sigma=0 & \text { dans } & \Omega \backslash\left(\mathcal{D} \cup \Gamma_{z} \cup \Gamma_{c}\right) \\
\sigma=A \varepsilon(u) & \text { dans } & \Omega \backslash\left(\mathcal{D} \cup \Gamma_{z} \cup \Gamma_{c}\right) \\
\sigma n=k \llbracket u_{2} \rrbracket n & \text { sur } & \Gamma_{z} \\
\sigma n=0 & \text { sur } & \partial \mathcal{D} \\
\sigma n=0 & \text { sur } & x_{1}= \pm L \\
\sigma n=\sigma_{\infty} n & \text { sur } & x_{2}= \pm L \\
\sigma n=\sigma_{c} n & \text { sur } & \Gamma_{c}
\end{array}\right.
$$

La loi gouvernant l'évolution de la pointe cohésive $\ell_{a}$ avec le chargement est donnée par:

$$
\llbracket u_{2}\left( \pm \ell_{a}\right) \rrbracket=\delta_{0}
$$

Cette phase cesse lorsque l'ouverture maximale dans la zone cohésive atteint la valeur critique $\delta_{c}$. La valeur de la charge correspondante est la charge de rupture $\sigma_{r}$;

3) phase de propagation. Une fois la charge de rupture atteinte, l'equilibre de la structure ne peut plus être assuré sans que se développe une zone de fissuration non 
cohésive. En tenant compte de la présence de cette zone, le champ des déplacements $u$ et le champ des contraintes $\sigma$ devront donc être solution du problème suivant :

$$
\left\{\begin{array}{ccc}
\text { div } \sigma=0 & \text { dans } & \Omega \backslash\left(\mathcal{D} \cup \Gamma_{z} \cup \Gamma_{c} \cup \Gamma_{n}\right) \\
\sigma=A \varepsilon(u) & \text { dans } & \Omega \backslash\left(\mathcal{D} \cup \Gamma_{z} \cup \Gamma_{c} \cup \Gamma_{n}\right) \\
\sigma n=k \llbracket u_{2} \rrbracket n & \text { sur } & \Gamma_{z} \\
\sigma n=0 & \text { sur } & \partial \mathcal{D} \cup \Gamma_{n} \\
\sigma n=0 & \text { sur } & x_{1}= \pm L \\
\sigma n=\sigma_{\infty} n & \text { sur } & x_{2}= \pm L \\
\sigma n=\sigma_{c} n & \text { sur } & \Gamma_{c}
\end{array} .\right.
$$

La loi gouvernant les évolutions de la pointe cohésive $\ell_{a}$ et de la pointe non cohésive $\ell_{c}$ avec le chargement est donnée par :

$$
\llbracket u_{2}\left( \pm \ell_{a}\right) \rrbracket=\delta_{0}, \quad \llbracket u_{2}\left( \pm \ell_{c}\right) \rrbracket=\delta_{c}
$$

\section{Résultats}

Plutôt que de faire diminuer la taille du défaut, nous avons choisi d'augmenter la longueur caractéristique $\delta_{c}$ tout en maintenant constante la contrainte critique $\sigma_{c}$ et la raideur de la phase élastique $\sigma_{c} / \delta_{0}$. Ce faisant on maintient constant le saut critique de décohésion $\delta_{0}$ et augmentons le rapport $\delta_{c} / \delta_{0}$. Ce choix n'est pas neutre et il serait intéressant de voir si les conclusions seraient les mêmes si l'on faisait un autre choix. Nous reportons cette étude à un travail futur.

Nous choisissons pour les dimensions du problème $L=100 \mathrm{~mm}, \ell_{0}=3 \mathrm{~mm}$. Pour les données matériau, on choisit celles d'un polymère PMMA

$$
\sigma_{c}=72 \mathrm{MPa}, E=3000 \mathrm{MPa}, \nu=0,36
$$

Enfin pour le saut critique de décohésion, nous choisissons $\delta_{0}=0,00402 \mathrm{~mm}$. Le domaine est maillé par des éléments finis triangulaires à 3 noeuds (pour des raisons de symétrie seul un quart du domine est maillé) figure 4. La ZCC est modélisée à l'aide d'éléments d'interface quadrangulaires d'épaisseur nulle.

\subsection{Phase élastique et charge d'amorçage}

On commence par faire un calcul purement élastique pour déterminer la charge d'amorçage $\sigma_{a}$, i.e. la charge $\sigma_{\infty}$ à partir de laquelle l'ouverture de la ZCC atteint le saut critique de décohésion $\delta_{0}$. Pour des raisons évidentes de symétrie et de conditions aux limites, l'ouverture maximale se situe au bord du défaut, aux points $\left(x_{1}= \pm \ell_{0}, x_{2}=0\right)$. La charge d'amorçage calculée est égale à 6,55 MPa pour la fissure et à 9,36 MPa pour le trou. 
488 Revue européenne de mécanique numérique. Volume 17 - n 4/2008

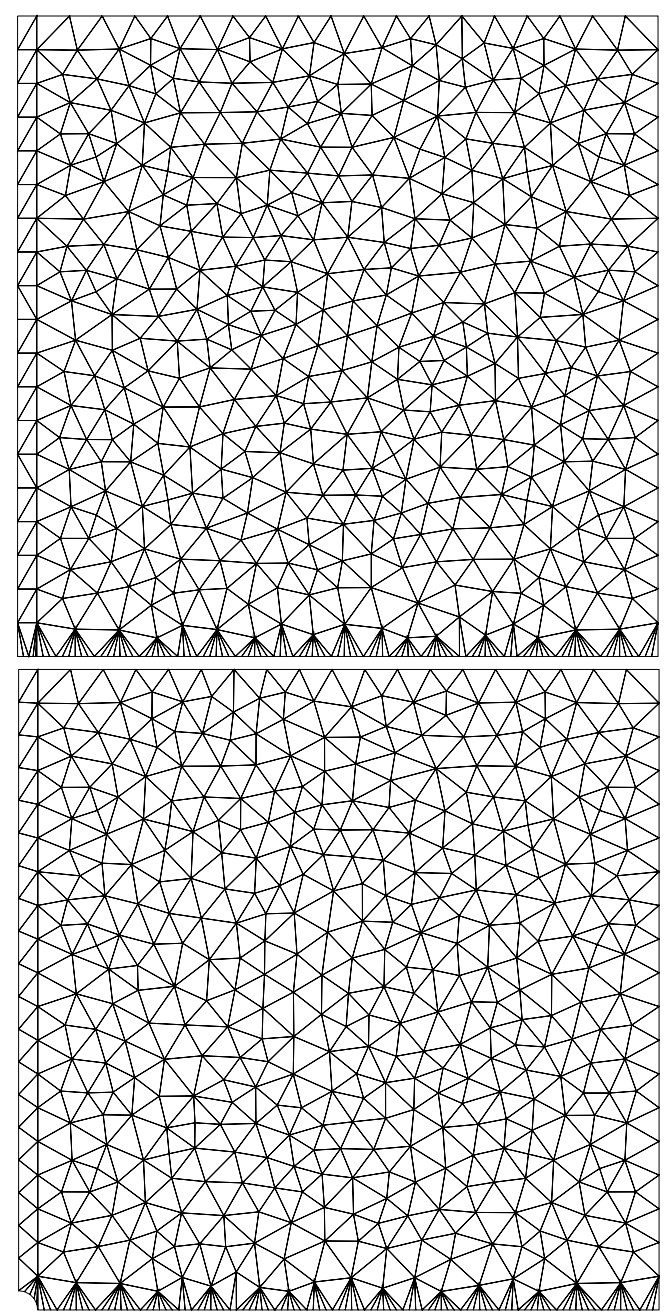

Figure 4. Maillage du quart du domine pour les cas du trou et de la fissure

\subsection{Phase d'amorçage}

Une zone cohésive apparaît et s'étend si l'on augmente la charge au-delà de $\sigma_{a}$. Le critère gouvernant la propagation de la pointe cohésive $\ell_{a}$ est $\llbracket u_{2}\left(\ell_{a}\right) \rrbracket=\delta_{0}$. C'est une relation implicite entre $\sigma_{\infty}$ et $\ell_{a}$. D'un point de vue pratique, il est plus facile de calculer $\sigma_{\infty}$ en supposant connue $\ell_{a}$. En effet, en utilisant la linéarité du problème élastique, l'ouverture $\llbracket u_{2}\left(\ell_{a}\right) \rrbracket$ peut s'écrire :

$$
\llbracket u_{2}\left(\ell_{a}\right) \rrbracket=\sigma_{\infty} \llbracket u_{2}\left(\ell_{a}\right) \rrbracket^{\infty}+\llbracket u_{2}\left(\ell_{a}\right) \rrbracket^{c}
$$


où $\llbracket u_{2}\left(\ell_{a}\right) \rrbracket^{\infty}$ et $\llbracket u_{2}\left(\ell_{a}\right) \rrbracket^{c}$ sont respectivement l'ouverture pour le problème sans forces cohésives et $\sigma_{\infty}=1$, et le problème avec forces cohésives et $\sigma_{\infty}=0$. L'équation $\llbracket u_{2}\left(\ell_{a}\right) \rrbracket=\delta_{0}$ donne :

$$
\sigma_{\infty}=\frac{\delta_{0}-\llbracket u_{2}\left(\ell_{a}\right) \rrbracket^{c}}{\llbracket u_{2}\left(\ell_{a}\right) \rrbracket^{\infty}}
$$

Donc, pour une valeur donnée de $\ell_{a}$, nous déterminons $\sigma_{\infty}$ par la formule [7]. Les résultats obtenus pour la fissure et le trou sont représentés sur la figure 5. On observe que le chargement $\sigma_{\infty}$ est une fonction croissante de la longueur de la zone cohésive. On observe également qu'à part la charge d'amorçage les courbes correspondant au trou et à la fissure sont quasiment identiques. On peut conclure que la sensibilité de la phase d'amorçage à la forme du défaut est faible.

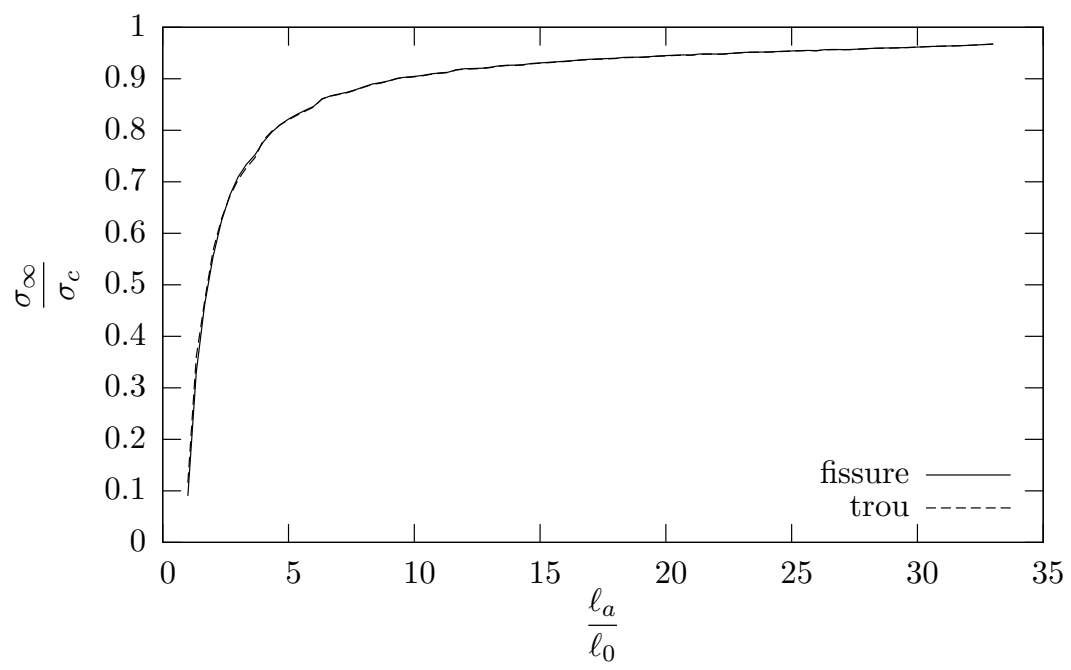

Figure 5. Relation entre la charge appliquée et la position de la zone cohésive dans la phase cohésive

\subsection{Charge de rupture}

La charge de rupture $\sigma_{r}$ est la contrainte appliquée correspondant à des ouvertures de la fissure $\llbracket u_{2}\left( \pm \ell_{0}\right) \rrbracket=\delta_{c}$ et $\llbracket u_{2}\left( \pm \ell_{a}\right) \rrbracket=\delta_{0}$. Pour déterminer la charge de rupture, pour une longueur caractéristique donnée, la méthode numérique suivante est utilisée. Pour une valeur testée de $\ell_{a}, \sigma_{\infty}$ est obtenue en résolvant l'équation $\llbracket u_{2}\left(\ell_{a}\right) \rrbracket=\delta_{0}$ selon la procédure expliquée dans la section 3.2. La bonne valeur de $\ell_{a}$ est obtenue par dichotomie de telle sorte que $\llbracket u_{2}\left(\ell_{0}\right) \rrbracket=\delta_{c}$. Nous calculons $\sigma_{r}$ pour plusieurs valeurs de la longueur caractéristique $\delta_{c}\left(\delta_{0}\right.$ restant fixe). 


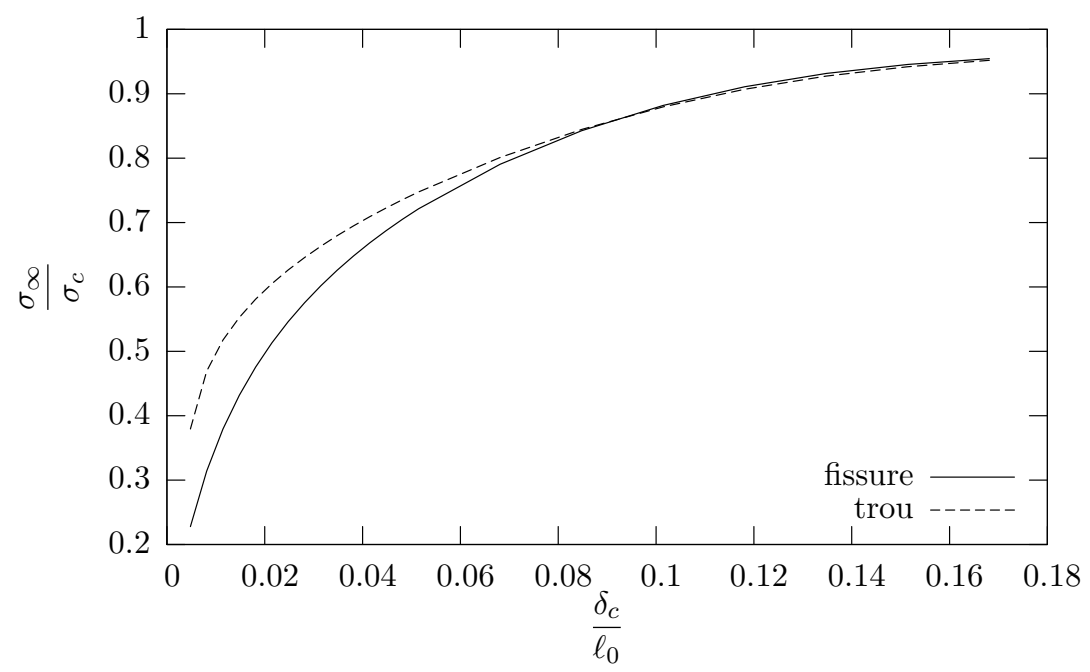

Figure 6. Relation entre la charge appliquée et la position de la zone cohésive dans la phase cohésive

Les valeurs numériques calculées pour la fissure et le trou sont représentées dans la figure 6 dans un diagramme $\sigma_{r}-\delta_{c}$. Comme attendu, la charge de rupture est une fonction croissante de $\delta_{c}$, mais on notera que la charge de rupture n'atteint jamais la valeur $\sigma_{c}$ quelle que soit la valeur de $\delta_{c}$. C'est dû au caractère fini des dimensions de la plaque. En effet, en utilisant un raisonnement élémentaire de calcul à la rupture, il est facile de voir que la plaque trouée ne peut pas supporter une charge plus grande que $\left(1-\frac{\ell_{0}}{L}\right) \sigma_{c}$ qui correspond à la charge d'équilibre de la plaque lorsque la zone cohésive occupe toute la largeur de la plaque. Compte tenu des valeurs numériques choisies, la charge de rupture ne peut donc dépasser 0,97 $\sigma_{c}$. Notons enfin que si l'on faisait tendre la longueur du défaut vers 0 ou la largeur de la plaque vers l'infini, alors la charge de rupture tendrait vers $\sigma_{c}$.

\subsection{Phase de propagation}

Il reste à prouver que $\sigma_{r}$ est réellement la charge maximale que la structure peut supporter tout en vérifiant les équations d'équilibre et les critères de propagation des différentes zones. Pour cela, on fixe la valeur de la pointe non cohésive $\ell_{c}$ et on calcule les valeurs de la charge $\sigma_{\infty}$ et de la pointe cohésive $\ell_{a}$ en résolvant le système d'équations non linéaires [5]. La méthode numérique utilisée est la suivante. Pour une valeur testée de $\ell_{a}, \sigma_{\infty}$ est obtenue en résolvant l'équation $\llbracket u_{2}\left(\ell_{a}\right) \rrbracket=\delta_{0}$ avec la procédure expliquée dans la section 3.2. La bonne valeur de $\ell_{a}$ est obtenue par dichotomie de telle sorte que $\llbracket u_{2}\left(\ell_{c}\right) \rrbracket=\delta_{c}$. 


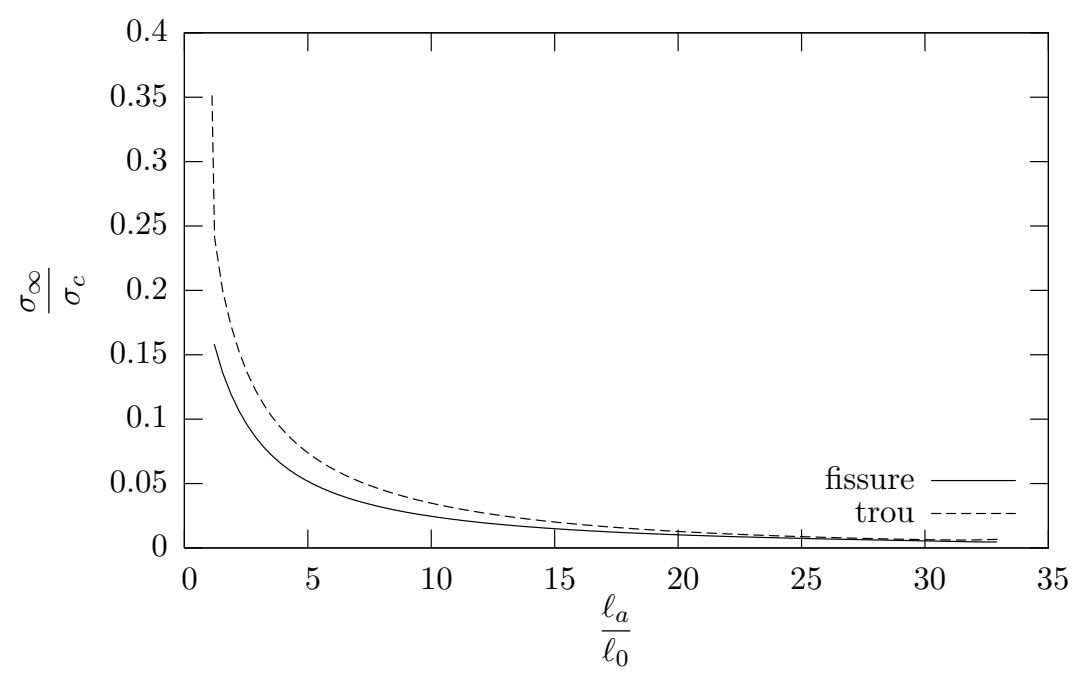

Figure 7. Relation entre la charge appliquée et la position de la zone cohésive dans la phase de propagation

Nous obtenons finalement $\sigma_{\infty}$ en fonction de $\ell_{a}$ pour un $\delta_{c}$ donné. Quand $\ell_{c}=\ell_{0}$ nous avons évidemment $\sigma_{\infty}=\sigma_{r}$. Il s'avère que $\sigma_{\infty}$ est une fonction décroissante de $\ell_{a}$, ceci prouve que $\sigma_{r}$ est une charge limite et par conséquent la charge de rupture de la structure. Par exemple, nous avons représenté sur la figure 7 la relation entre $\sigma_{\infty}$ et $\ell_{a}$ pour une valeur de $\delta_{c}$ égale à $0,00804 \mathrm{~mm}$ respectivement pour le trou et la fissure. Les charges de rupture sont $0,35 \sigma_{c}$ pour le trou et $0,16 \sigma_{c}$ pour la fissure.

\section{Conclusion générale}

En comparant les réponses obtenues pour la plaque fissurée et la plaque trouée, on voit que la charge d'amorçage est sensible à la forme du défaut, mais pas à sa taille. Par contre, la charge limite de la structure est très sensible à la taille du défaut, mais peu à sa forme. De plus, on voit que plus le défaut est petit, la longueur de référence étant la longueur caractéristique du matériau introduite dans le modèle des forces cohésives, plus la charge limite se rapproche de la contrainte critique du matériau. Autrement dit, ceci semble indiquer que les défauts de petite taille ont peu d'influence sur les capacités de résistance d'une structure si l'on adopte le modèle des forces cohésives. Ce résultat est identique au résultat obtenu par Ferdjani et al. (2006a), Ferdjani et al. (2006b) et Ferdjani et al. (2007) dans le cas du modèle de Dugdale. Il serait évidemment intéressant de généraliser ce résultat à n'importe quel type de défaut, n'importe quel type de structure et n'importe quel modèle de force cohésive. Cela nécessitera 
le développement d'outils théoriques et numériques adaptés. Mais on peut considérer que les deux cas traités, extrêmes de par la forme du défaut choisie, et les deux modèles de forces cohésives étudiés sont un gage du caractère générique du résultat.

\section{Remerciements}

Ce travail a été partiellement effectué avec le soutien financier de l'OTAN dans le cadre de l'accord PST.MD.CLG 980960 et du CNRS dans le cadre d'un accord de coopération DEF/CNRS 02DZA1079.

\section{Bibliographie}

Budiansky B., Evans A. G., Hutchinson J. W., "Fiber-matrix debonding effects oncracking in aligned fiber ceramic composites", Int. J. Solids Structures, vol. 32, n 3-4, 1995, p. 315328.

Chaboche J.-L., Feyel F., Monerie Y., "Interface debonding models : a viscous regularization with a limited rate dependency", Int. J. Solids Structures, vol. 38, n 18, 2001, p. 3127-3160.

Charlotte M., Francfort G., Marigo J.-J., Truskinovsky L., "Revisiting brittle fracture as an energy minimization problem : comparison of Griffith and Barenblatt surface energy models.", in A. Benallal (ed.), Proceedings of the Symposium on Continuous Damage and Fracture, Elsevier, Paris, 2000, p. 7-12.

Charlotte M., Laverne J., Marigo J.-J., "Initiation of cracks with cohesive force models : a variational approach", Eur. J. Mech. A/Solids, vol. 25, n 4, 2006, p. 649-669.

Del Piero G., "One-dimensional ductile-brittle transition, yielding, and structured deformations", Variations of domain and free-boundary problems in solid mechanics (Paris, 1997), vol. 66 of Solid Mech. Appl., Kluwer Acad. Publ., Dordrecht, 1999, p. 203-210.

Del Piero G., Truskinovsky L., "Macro- and micro-cracking in one-dimensional elasticity", Int. J. Solids Structures, vol. 38, n 6, 2001, p. 1135-1138.

Dugdale D. S., "Yielding of steel sheets containing slits", J. Mech. Phys. Solids, vol. 8, n², 1960, p. 100-108.

Ferdjani H., Abdelmoula R., Marigo J.-J., « Etude de l'influence des défauts de petite taille sur le comportement à rupture avec le modèle de Dugdale », Revue Européenne de Mécanique Numérique, vol. 15, n 4, 2006a, p. 409-425.

Ferdjani H., Abdelmoula R., Marigo J.-J., "Study of the influence of small size defects on the rupture behavior with the Dugdale model.", Third International Conference on Advances in Mechanical Engineering and Mechanics, 2006b.

Ferdjani H., Abdelmoula R., Marigo J.-J., "Insensitivity to small defects of the rupture of matarials governed by the Dugdale model", Contin. Mech. Thermodyn., vol. 19, 2007, p. 191210.

Laverne J., Marigo J.-J., « Approche globale, minima relatifs et Critère d'Amorçage en Mécanique de la Rupture », Comptes Rendus Mécanique, vol. 332, n 4, 2004, p. 313-318.

Needleman A., "A continuum model for void nucleation by inclusion debonding", J. Appl. Mech., vol. 54, 1987, p. 525-531. 
Needleman A., "An analysis of tensile decohesion along an interface", J. Mech. Phys. Solids, vol. 38, n 3, 1990, p. 289-324.

Needleman A., "Micromechanical modelling of interface decohesion", Ultramicroscopy, vol. 40, 1992, p. 203-214.

Nguyen O., Repetto E. A., Ortiz M., Radovitzki R. A., "A cohesive model of fatigue crack growth", Int. J. Frac. Mech., vol. 110, n 4, 2001, p. 351-369.

Roe K. L., Siegmund T., "An irreversible cohesive zone model for interface fatigue crack growth simulation", Engineering Frac. Mech., vol. 70, n 2, 2002, p. 209-232.

Tvergaard V., "Effect of fiber debonding in a whisker-reinforced metal", Mat. Sci. Eng. A-Struc., vol. 125, 1990, p. 203-213.

Xie D., Waas A., "Discrete cohesive zone model for mixed-mode fracture using finite element analysis", Engineering Frac. Mech., vol. 73, n 13, 2006, p. 1783-1796. 
